\title{
Differential Regulation of the Promoter Activity of the Mouse UCP2 and UCP3 Genes by MyoD and Myogenin
}

\author{
Dongho Kim ${ }^{1, *}$, Sarawut Jitrapakdee ${ }^{2}$ and Mary Thompson ${ }^{1}$ \\ ${ }^{1}$ Department of Biochemistry, University of Otago, PO Box 56, Dunedin 9054, New Zealand \\ ${ }^{2}$ Department of Biochemistry, Faculty of Science, Mahidol University, Bangkok 10400, Thailand
}

Received 24 April 2007, Accepted 4 July 2007

\begin{abstract}
UCP2 and UCP3 are members of the uncoupling protein family, which may play roles in energy homeostasis. In order to determine the regulation of the predominant expression of UCP3 in skeletal muscle, the effects of differentiation and myogenic regulatory factors on the promoter activities of the mouse UCP2 and UCP3 genes were studied. Reporter plasmids, containing approximately $3 \mathrm{~kb}$ of the 5'-upstream region of the mouse UCP2 and UCP3 genes, were transfected into $\mathrm{C} 2 \mathrm{C} 12$ myoblasts, which were then induced to differentiate. Differentiation positively induced the reporter expression about 20 -fold via the UCP3 promoter, but by only 2 -fold via the UCP2 promoter. $\mathrm{C} 2 \mathrm{C} 12$ myoblasts were cotransfected with expression vectors for myogenin and/or MyoD as well as reporter constructs. The simultaneous expression of myogenin and MyoD caused an additional 20 -fold increase in the reporter expression via the UCP3 promoter, but only a weak effect via the UCP2 promoter. In L6 myoblasts, only MyoD activated the UCP3 promoter, but in 3T3-L1 cells neither factor activated the UCP3 promoter, indicating that additional cofactors are required, which are present only in $\mathrm{C2C12}$ myoblasts. The expression of $\mathrm{UCP} 2$ and UCP3 is differentially regulated during muscle differentiation due to the different responsiveness of their promoter regions to myogenin and MyoD.
\end{abstract}

Keywords: MyoD, Myogenin, Promoter activity, UCP2, UCP3

\footnotetext{
*To whom correspondence should be addressed.

Tel: 64-3-479-7876; Fax: 64-3-479-7866

E-mail: dongho.kim@stonebow.otago.ac.nz
}

\section{Introduction}

Uncoupling proteins (UCP)-2 and -3 are members of the uncoupling protein family of mitochondrial inner membrane proteins (Krauss et al., 2005), which share 59 and 57\% amino acid identity with UCP1, respectively. UCP1 is a protein with a known thermogenic function in brown adipose tissue; UCP2 and UCP3 have also been shown to act as uncouplers of oxidative phosphorylation (Marti et al., 2001; Horvath et al., 2003). Variations in the activity or regulation of UCP2 or UCP3 may contribute to obesity and its associated disorders by altering energy homeostasis. However, UCP2 and UCP3 do not appear to be solely involved in thermogenesis, but also have proposed roles in modulating the generation of reactive oxygen species (Arsenijevic et al., 2000; Vidal-Puig et al., 2000) and in lipid handling (Himms-Hagen and Haper, 2001; Schrauwen et al., 2003). Although the physiological roles of UCP2 and UCP3 still remain controversial, a better understanding of their function and regulation may have implications in the future management of many pathological conditions, including obesity and diabetes.

The expressions of UCP2 and UCP3 have different tissue distributions, which may give clues as to their function. UCP2 is expressed in many tissues; whereas, UCP3 is expressed preferentially in skeletal muscle. Although accurate comparison is difficult, UCP3 mRNA appears to be expressed at higher levels than that of UCP2 in the skeletal muscle in rodents (Boss et al., 1997; Matsuda et al., 1997; Vidal-Puig et al., 1997; Weigle et al., 1998; Yoshitomi et al., 1998) and humans (Boss et al., 1997; Larkin et al., 1997; Vidal-Puig et al., 1997; Chevillotte et al., 2001). UCP2 and UCP3 gene expressions are highly sensitive to the metabolic status, and are upregulated in a number of physiological situations, in a tissuedependent manner (Thompson and Kim, 2004). Skeletal muscle is an important site of glucose and lipid metabolism related to energy homeostasis; therefore, it is of interest to determine the regulation of the expression of UCP3 in this type of tissue. 
In cultured muscle cells the UCP3 mRNA levels are characteristically very low (Hwang and Lane, 1999; Solanes et al., 2000). The expression of UCP3 is induced upon differentiation of myoblasts to myotubes (Shimokawa et al., 1998; Nagase et al., 1999; Kim, 2000; Solanes et al., 2000; Son et al., 2001); whereas, UCP2 is already expressed in myoblasts and its mRNA levels increase with much less magnitude upon differentiation to myotubes (Shimokawa et al., 1998). Differentiation of skeletal muscle cells, and the subsequent expression of myotube-specific genes, is under the control of a number of myogenic factors, including myoD and myogenin (Weintraub, 1993). The aim of this study was to determine whether differentiation and myogenic regulatory factors had different affects on the activity of the promoter regions of the UCP2 and UCP3 genes and; therefore, contribute to the predominant expression of UCP3 in skeletal muscle.

\section{Materials and methods}

Cell lines. The mouse muscle cell line, $\mathrm{C} 2 \mathrm{C} 12$, the rat muscle cell line, L6, and the mouse preadipocyte cell line, 3T3-L1, were obtained from the American Type Culture Collection.

Plasmids. pGEM-T Easy, pGL3-Basic firefly luciferase reporter and pRL-TK Renilla luciferase reporter vectors were from Promega. pVenus, a yellow fluorescence protein expression vector (Nagai et al., 2002), was provided by Dr. Brown (University of Otago). Expression vectors driven by Moloney murine leukemia virus LTR for mouse MyoD and myogenin were provided by Dr. Koishi (University of Otago).

Plasmid construction. Primer Express 1.5 was used to design gene specific primers based on the Genbank database for UCP2 (Accession numbers: $\underline{\mathbf{A F 1 1 5 3 1 9}}$ (forward primers) and $\underline{\mathbf{A F 0 9 6 2 8 8}}$ (reverse primers)) and UCP3 (Accession number: AB010742). Amplification of an approximate $3 \mathrm{~kb}$ fragment of the 5 '-upstream region of the UCP3 gene was performed using mouse genomic DNA libraries within GenomeWalker, Clontech, according to manufacturer's instructions, employing the gene-specific primers, GSP1 5'-CTGTGCGTCTAGCCAAGGTTGGGTAGT-3' and GSP2 5'-GGGTAGTGCAGGGCCACCCTAAGG-3'. An approximate $3 \mathrm{~kb}$ fragment of the 5'-upstream region of mouse UCP2 gene was amplified from mouse genomic DNA (approximately $500 \mathrm{ng}$ ) employing the forward and reverse primers 5'-TTGTGGCAAAA GCATGGCGCACTG-3' and 5'-GTGTCCGATGGCGGGAGAGT GGTG-3', respectively, for primary PCR, and 5'-CCGCTGTGTAG CTTTATGCGGGAATCAC-3' and 5'-CGACGACACCGAGGGC GGAACTGAC-3', respectively, for secondary PCR, using the High Fidelity PCR System, Roche, according to the manufacturer's instructions. The products were A-tailed and inserted into the pGEM-T Easy vector, according to the manufacturer's instructions. The purified pGEM-T Easy plasmids, containing the 5'-region of UCP2 and UCP3, were used as templates for the amplification of an approximate 3,000 bp fragment of each region. The forward and reverse primers included extensions to generate $K p n I$ and NheI restriction sites at its 5 ' end, respectively: The forward and reverse primers, 5'-CGGGGTACCGTGTAGCTTTATGCGGGAATC-3' and 5'CGGTGCTAGCAGGGCGGAACTGACAGTAGCTG-3', for UCP2; 5'-CGGGGTACCGCTGGTATTGGAAGAAACATGG-3' and 5'CGGTGCTAGCTAGTGCAGGGCCACCCTAAG-3' for UCP3. The purified PCR products were each digested with $K p n \mathrm{I}$ and NheI, purified and ligated into the KpnI-NheI restricted pGL3-Basic firefly luciferase reporter plasmid and transformed into DH5a cells, with positive clones then selected. The reporter constructs, pGL3Ucp2 and pGL3-Ucp3, were confirmed by sequencing.

Transient transfection and luciferase assay. Cells were cultured in 6-well plates using Dulbecco's modified Eagle's medium (DMEM; high glucose, $25 \mathrm{mM}$ ), supplemented with $10 \%$ fetal calf serum and $40 \mathrm{mg} / \mathrm{L}$ gentamycin, at $\mathrm{pH} 7.4$ in an humidified $95 \%$ air: $5 \% \mathrm{CO}_{2}$ atmosphere. Transfection was carried out when the cells reached $70 \%$ confluence, using FuGENE 6 (Roche), with a ratio of FuGENE $6(\mathrm{~mL})$ to DNA $(\mathrm{mg})$ of $3: 1$. The cells from each well were transfected with $500 \mathrm{ng}$ of the pGL3 reporter vector, 150 ng of the mammalian expression vectors for MyoD and/or myogenin and $50 \mathrm{ng}$ of pVenus or pRL-TK (Promega) as internal controls, in a total volume of $100 \mu \mathrm{L}$ DMEM. For standard promoter activity experiments, transfected cells were incubated for $48 \mathrm{~h}$, and then harvested for use in reporter gene assays. To test the effect of differentiation, $\mathrm{C} 2 \mathrm{C} 12$ myoblasts at about $70 \%$ confluence were transfected with the pGL3-Basic, pGL3-Ucp2 or pGL3-Ucp3 and pVenus vectors as an internal control. Two days later, when cells had reached $100 \%$ confluence, their differentiation was induced by replacing the medium with DMEM containing $2 \%$ fetal calf serum, with this point designated as day 0 . The first sample was harvested on day 0 , with subsequent samples harvested each day until day 3 .

Cells were lysed with $1 \times$ Passive Lysis Buffer (Promega), and the supernatant assayed for reporter gene activity. The firefly and Renilla luciferase activities were measured using the Dual-Luciferase, Reporter Assay System (Promega) employing an AutoLumat LB953 luminometer (Berthold Technologies, Bad Wildbad, Germany). The Venus expression was measured from the fluorescence using a POLARstar OPTIMA fluorometer (BMG Labtech, Offenburg, Germany), with excitation and emission wavelengths of 485 and $520 \mathrm{~nm}$, respectively. The firefly luciferase activity from triplicate determinations was normalised to that of the appropriate control, Renilla luciferase activity or the fluorescence value of Venus, of the corresponding sample. Statistically significant differences between the control and experimental groups were determined using the Student's $t$-test.

\section{Results}

Basal promoter activity of cloned 5'-upstream region of mouse UCP2 and UCP3. The 5'-upstream regions of mouse UCP2 (3,069 bp) and UCP3 (3,182 bp) were cloned and inserted into a pGL3-Basic firefly luciferase plasmid for the construction of the reporter plasmid pGL-Ucp2 and pGLUcp3, respectively. The basal promoter activities of the cloned regions were analysed in two mammalian muscle cell lines, C2C12 and L6, and a preadipocyte cell line, 3T3-L1 (Fig. 1). 


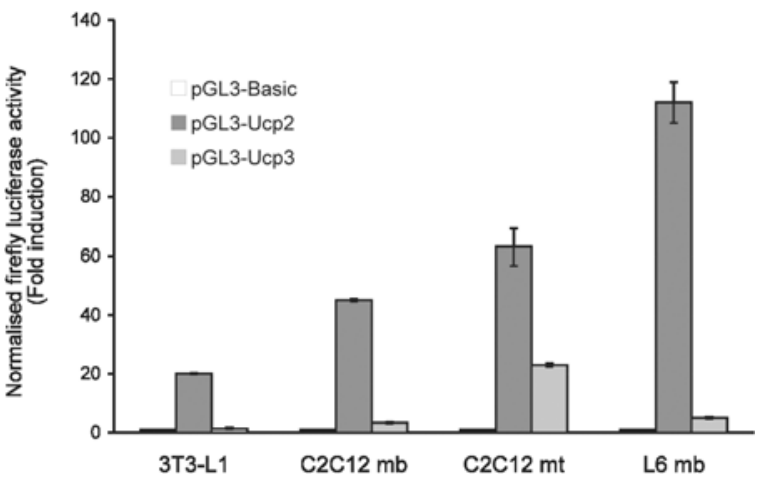

Fig. 1. Basal promoter activities of the cloned 5'-upstream regions of the UCP2 and UCP3 genes. Cells at $70 \%$ confluence were transfected with either pGL3-Basic, pGL3-Ucp2 or pGL3-Ucp3 and pRL-TK, harvested $48 \mathrm{~h}$ later and the luciferase activity measured. The differentiation of transfected $\mathrm{C} 2 \mathrm{C} 12$ myoblasts was induced at $100 \%$ confluence, and the cells harvested 3 days later. The firefly luciferase activity was normalised to that of Renilla luciferase. The bars represent the means $\pm \mathrm{SD}$ of triplicate determinations of the fold inductions of pGL3-Ucp2 and pGL3Ucp3 compared to pGL3-Basic, which was set at 1.0. 3T3-L1, mouse preadipocytes; $\mathrm{C} 2 \mathrm{C} 12 \mathrm{mb}$, mouse myoblasts; $\mathrm{C} 2 \mathrm{C} 12 \mathrm{mt}$, mouse myotubes; L6 mb, rat myoblasts.

The 5'-upstream region of the UCP2 gene induced 20-, 45and 112-fold increases in the firefly luciferase activities in the undifferentiated cell lines, 3T3-L1, C2C12 and L6, respectively, compared to that produced by the promoterless pGL3-Basic. In contrast, the 5'-upstream region of the UCP3 gene only induced 5-fold increases in the luciferase activities in $\mathrm{C} 2 \mathrm{C} 12$ and L6 cells, but the promoter activity was almost nonexistent in 3T3-L1 cells (1.4-fold). This difference in the promoter activities between UCP2 and UCP3 suggests the promoter of UCP2 was constitutively active; whereas, additional factors were needed to activate the UCP3 promoter.

When transfected $\mathrm{C} 2 \mathrm{C} 12$ myoblasts were induced to differentiate into myotubes, the promoter activity of pGL3Ucp3 increased 7-fold, from 3.2- to 23-fold above that of pGL3-Basic, whereas that of pGL3-Ucp2 only increased slightly, by 1.4-fold, from 45- to 63-fold (Fig. 1). However, the total time taken for expression of the reporter gene for both pGL3-Ucp2 and pGL3-Ucp3 was longer in C2C12 myotubes than in $\mathrm{C} 2 \mathrm{C} 12$ myoblasts. This longer incubation period for the $\mathrm{C} 2 \mathrm{C} 12$ myotubes would generate an accumulative expression of the reporter gene, which could consequently generate an apparently higher induction of firefly luciferase activity in $\mathrm{C} 2 \mathrm{C} 12$ myotubes. Nevertheless, these results suggest that differentiation could influence the expression of the reporter gene to a greater extent via the cloned 5'-upstream region of the UCP3 gene than via that of the UCP2 gene.

Effect of muscle cell differentiation on promoter activity. To determine the time course of the effects of the differentiation of $\mathrm{C} 2 \mathrm{C} 12$ myoblasts to myotubes on the promoter activities of

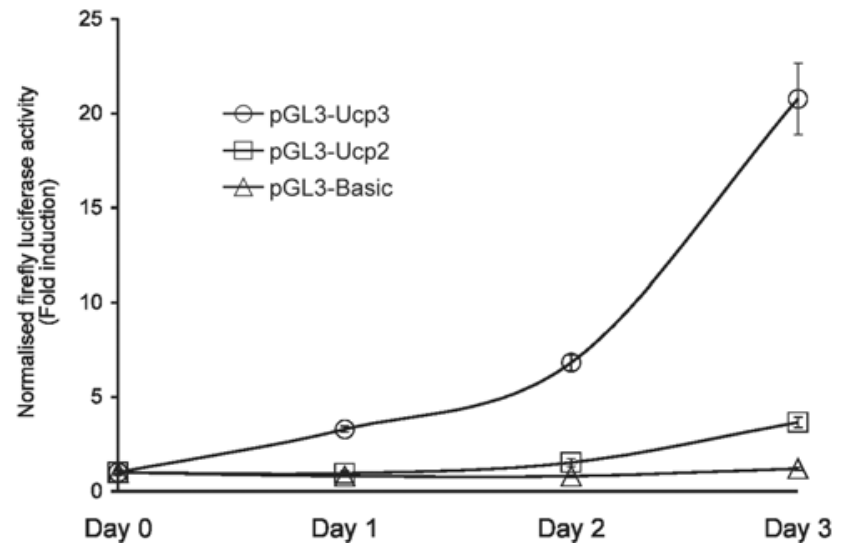

Fig. 2. Changes in the promoter activity during $\mathrm{C} 2 \mathrm{C} 12$ myoblast differentiation. $\mathrm{C} 2 \mathrm{C} 12$ myoblasts were transfected with either pGL3-Basic, pGL3-Ucp2 or pGL3-Ucp3 and pVenus. At confluence, differentiation of the transfected cells was induced (day 0 ), with the cells cultured until day 3. Groups were harvested on days $0,1,2$ and 3, with luciferase assays and fluorescence measurements then performed. In each group, the normalised firefly luciferase activities on days 1,2 and 3 were compared with that on day 0 , with the fold inductions determined. The values on each day are the means $\pm \mathrm{SD}$ of triplicate determinations.

UCP2 and UCP3, experiments were designed to take account of the different incubation times since transfection. The absolute reporter gene activities of pGL3-Basic, pGL3-Ucp2 and pGL3-Ucp3 from that of the internal control (pVenus) increased from days 0 to 3 . To account for the accumulative changes in expression during $\mathrm{C} 2 \mathrm{C} 12$ myoblast differentiation, the firefly luciferase activities from samples harvested on each day were normalised to that of the fluorescence value of the internal control. These normalised values for each group were compared relative to day 0 , with the fold induction on each day during myoblast differentiation then calculated. There was no change in the normalised luciferase activity of pGL3basic on each day throughout differentiation (Fig. 2), indicating that the observed increase in the absolute activities of both firefly luciferase and the internal control were most likely due to an accumulative increase due to the longer incubation time. In the case of pGL3-Ucp2, the results were similar to those of pGL3-Basic, although a 2-fold induction of normalised firefly luciferase activity was observed by day 3 (Fig. 2). In contrast, the fold induction of the normalised luciferase activity of pGL3-Ucp3 increased each day, with a 20 -fold increase observed compared to that at day 0 (Fig. 2). These results indicate that the differentiation of $\mathrm{C} 2 \mathrm{C} 12$ myoblasts had a positive affect on the expression of the reporter gene via the cloned UCP3 promoter region, and that the UCP2 and UCP3 genes are regulated in different manners during muscle differentiation.

Effect of myogenin and MyoD on promoter activity. The positive effect of muscle differentiation on the expression of 


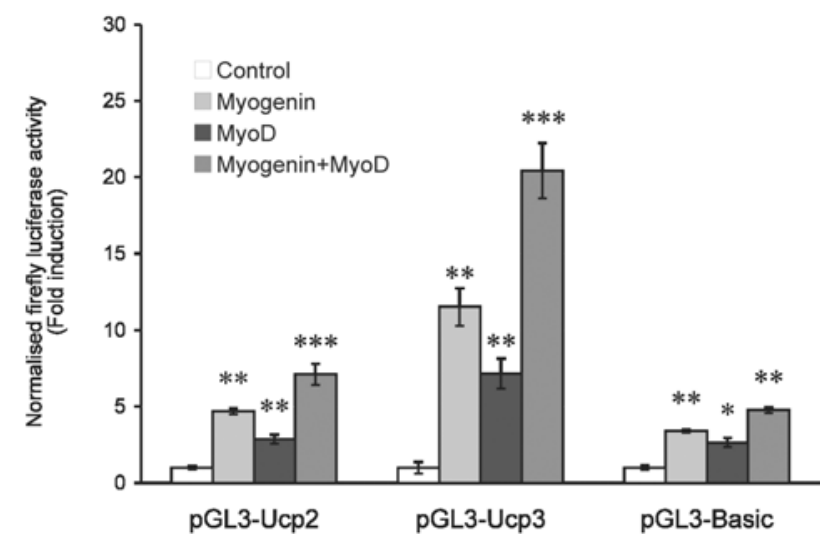

Fig. 3. The effects of myogenin and $\mathrm{MyoD}$ on the activities of the $\mathrm{UCP} 2$ and $\mathrm{UCP} 3$ promoters in $\mathrm{C} 2 \mathrm{C} 12$ myoblasts. $\mathrm{C} 2 \mathrm{C} 12$ myoblasts were transfected with the reporter gene constructs, pGL3-Ucp2, pGL3-Ucp3 or pGL3-Basic, and the internal control, pVenus, in the presence or absence of the myogenin or MyoD expression vector. After $48 \mathrm{~h}$, the cells were harvested for reporter gene assays. The normalised firefly luciferase activity of the experimental group was compared to that of the control group, which was transfected with an empty expression vector containing no cDNA. The control group (open bar) value was set at 1.0, with the fold induction of each group determined. The bars represent the means $\pm \mathrm{SD}$ of triplicate determinations. Significant differences between the control and cotransfected groups are marked with asterisks: *, $p<0.05 ; * *, p<0.005 ; * * *, p<0.001$.

the reporter gene via the UCP3 promoter region suggested regulation by myogenic regulatory factors, similarly to the promoters of other muscle-specific genes. To test for a possible role of myogenic regulatory factors in the regulation of UCP2 and UCP3 gene expression, myogenin and MyoD were selected as the most likely transcription factors to be involved, based on their mRNA expression patterns during C2C12 myoblast differentiation (Shimokawa et al., 1998; Dedieu et al., 2002). Proliferating C2C12 myoblasts were cotransfected with the myogenic regulatory factor expression vectors or the control vector and each reporter gene construct. After $48 \mathrm{~h}$, the cells were harvested, with reporter gene assays performed, as described in the Methods, the results of which are shown in Fig. 3.

In the pGL3-Ucp3 transfected group, the co-expression of myogenin or MyoD alone caused 11- and 7-fold increases in the luciferase activity, respectively. The simultaneous expression of myogenin and MyoD caused an additional 20-fold increase in the luciferase activity. Although the pGL3-Basic vector showed responsiveness to both myogenin and $\mathrm{MyoD}$, for pGL3-Ucp3 the activity was increased 3.3-fold more by myogenin, 2.6-fold more by MyoD and 4-fold more by both combined, compared to the corresponding activities in cells transfected with pGL3-Basic, indicating the increases in the luciferase activity brought about by to these two transcription factors was via the cloned UCP3 promoter region of the pGL3-Ucp3 vector.

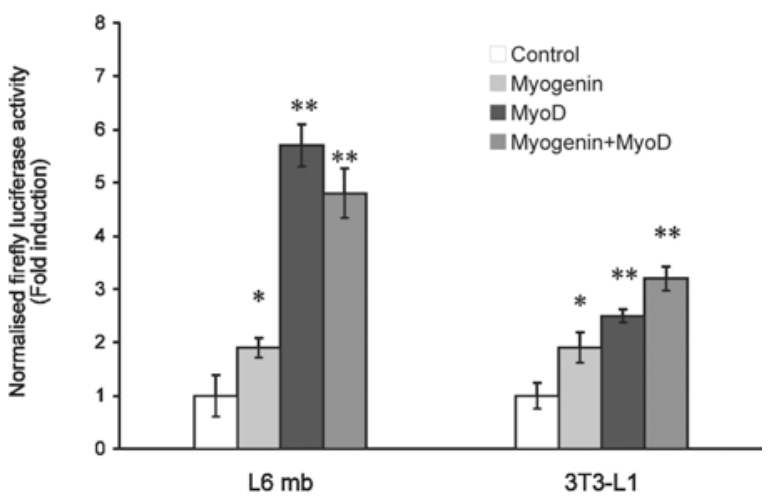

Fig. 4. The effects of myogenin and MyoD on the UCP3 promoter activity in different cell lines. L6 myoblasts and 3T3-L1 preadipocytes were cotransfected with pGL3-Ucp3 and MyoD and/or myogenin expression vectors. As a control group, an empty expression vector containing no cDNA was transfected. All cells were also transfected with pVenus as internal controls. After $48 \mathrm{~h}$, the cells were harvested for reporter gene assays. The normalised firefly luciferase activities of each group were compared with that of the control group. The control group (open bar) value was set at 1.0, with fold induction of each group plotted. Bars represent the means \pm SD of triplicate determinations. Significant differences between the control and cotransfected groups are marked with asterisks: *, $p<0.05 ; * *, p<0.01$.

In contrast, for the pGL3-Ucp2 transfected group, the coexpression of myogenin and MyoD resulted in only 4.7- and 2.9-fold inductions of luciferase activity, respectively, via the cloned UCP2 promoter region. The simultaneous expression of myogenin and MyoD caused a 7-fold increase in the luciferase activity. However, the induction of luciferase by MyoD was almost the same as the corresponding promoterless control, but the induction by myogenin and both the myogenic factors together was only 1.4-fold greater than the corresponding promoterless control. These results indicate the cloned UCP2 promoter responds only weakly, if at all, to both myogenin and MyoD.

To find if both myogenin and $\mathrm{MyoD}$ are required and sufficient for transactivation of the UCP3 promoter, the effects of the co-expression of myogenin and/or MyoD on the activation of the UCP3 promoter region were investigated in other cell lines. In L6 myoblasts, another rodent skeletal muscle cell line, which is also capable of undergoing differentiation, the co-expression of MyoD caused the greatest activation of the UCP3 promoter (by 6-fold); whereas, the effect of myogenin was not significant (Fig. 4). When both myogenin and MyoD were transfected, the luciferase activity increased no further, but remained at similar levels to that caused by MyoD alone, indicating no additive interaction of myogenin and MyoD. These results are in contrast to the observations in $\mathrm{C} 2 \mathrm{C} 12$ myoblasts (Fig. 3), suggesting the interactions of these two transcription factors may be different in $\mathrm{C} 2 \mathrm{C} 12$ and L6 myoblasts. 
In 3T3-L1 cells, a mouse preadipocyte cell line, myogenin and MyoD each had only a minimal effect, if any, on the induction of luciferase from the UCP3 promoter region, although the simultaneous expression of both myogenin and MyoD did improve the relatively low induction of luciferase by 3.2-fold (Fig. 4). The magnitude of the effect was nowhere near as great as that seen in $\mathrm{C} 2 \mathrm{C} 12$ cells.

\section{Discussion}

The cloned 5'-upstream regions of the mouse UCP2 and UCP3 genes showed varying degrees of promoter activity when transfected into various cultured cell lines. The activity of the UCP2 promoter region was about 10-fold greater than the UCP3 promoter in all the cell types studied, suggesting it was constitutively active, which was consistent with the observation that endogenous UCP2 is expressed in many different tissues, including adipose tissue and skeletal muscle (Boss et al., 1997; Matsuda et al., 1997; Vidal-Puig et al., 1997; Yoshitomi et al., 1998). Conversely, the activity of the UCP3 promoter region was almost undetectable in undifferentiated cells. However, its activity increased 20-fold during differentiation of the mouse skeletal muscle cell line, $\mathrm{C} 2 \mathrm{C} 12$, into myotubes. The UCP3 promoter region was activated in a manner proportional to the state of differentiation. In contrast, there was no significant difference in the activity of the UCP2 promoter region between myoblasts and myotubes, although the absolute activity of the UCP2 promoter region was always higher than that of UCP3. This pattern of promoter activity reflects the preferential expression of UCP3 in skeletal muscle (Boss et al., 1997; Larkin et al., 1997; Matsuda et al., 1997; Vidal-Puig et al., 1997; Weigle et al., 1998; Yoshitomi et al., 1998; Chevillotte et al., 2001), which closely resembles those of the expression of endogenous UCP2 and UCP3 during C2C12 differentiation (Shimokawa et al., 1998; Kim, 2000; Solanes et al., 2000). In the rat skeletal muscle cell line, L6, a similar differentiation-dependent expression of endogenous UCP3 mRNA has been observed (Nagase et al., 1999; Solanes et al., 2000; Son et al., 2001).

The present study has shown that both myogenin and MyoD contribute to the up-regulation of the UCP3 expression in $\mathrm{C} 2 \mathrm{C} 12$ myoblasts. The forced expressions of the myogenic regulatory transcription factors, MyoD and myogenin, in undifferentiated $\mathrm{C} 2 \mathrm{C} 12$ myoblasts was conducted to mimic the differentiated state. Both myogenin and MyoD alone induced significant activation of the UCP3 promoter region, and had an additive effect on the reporter gene expression when in combination via the UCP3 promoter region in $\mathrm{C} 2 \mathrm{C} 12$ myoblasts, to a level similar to that seen in differentiated C2C12 myotubes. Conversely, these myogenic factors had only minimal effects on the UCP2 promoter region. The greater responsiveness of the UCP3 promoter region to myogenin and MyoD compared to that of UCP2 was consistent with the more marked increase in the reporter gene activity seen with pGL3-Ucp3 during C2C12 differentiation. Myogenin and MyoD are most likely important regulators of expression of the UCP3 gene during muscle differentiation. Further studies will be required to show that these transcription factors bind directly to the UCP3 promoter.

Interestingly, the effects of MyoD and myogenin were different in another muscle cell line, L6 myoblasts; MyoD increased the reporter activity, but myogenin had no effect, but the induction when in combination was no greater than for MyoD alone. Other investigations have also shown that in L6 myoblasts MyoD is required to activate the promoter of human UCP3 (Solanes et al., 2000; Solanes et al., 2003) and; more recently, mouse UCP3 (Solanes et al., 2005; Pedraza et al., 2006), which is consistent with the present results; however, the effect of myogenin was not tested. The different levels of endogenous MyoD between C2C12 and L6 cells (Wright et al., 1989; Shimokawa et al., 1998; Dedieu et al., 2002) could affect the control levels of promoter activity and; thus, contribute to the observed cell line-specific activity of the UCP3 promoter region. However, the involvement of other coactivators for fully functional MyoD and myogenin is more likely.

Additional factors appear to be required, as in the nonskeletal muscle cell line, 3T3-L1 preadipocytes, the forced expression of MyoD and myogenin did not induce activation of the UCP3 promoter. Taken together, these results indicate that both MyoD and myogenin are necessary, but are not sufficient by themselves to induce UCP3 promoter activity. Therefore, additional, as yet unidentified trans-acting cofactors, which are present in $\mathrm{C} 2 \mathrm{C} 12$ myoblasts but not in 3T3-L1 cells, are also required.

Myogenic regulatory factors, which are characterised by a basic region and adjacent helix-loop-helix motif, readily form heterodimers with other ubiquitous helix-loop-helix proteins of the E protein class (Murre et al., 1989). The binding sequence for transcription factors containing the basic helixloop-helix motif is CANNTG, known as an E-box. Recently, a novel non-canonical $\mathrm{E}$ box in the proximal region to the transcription initiation site of human UCP3 has been functionally identified as the site of action of MyoD (Solanes et al., 2003). It is not known whether a similar functional noncanonical E box is present in the mouse UCP3 promoter region. Computer analysis has found potential transcription regulatory motifs for the binding of both myogenin and MyoD in the promoter regions of both UCP2 (Yoshitomi et al., 1999; Kim, 2006) and UCP3 (Yoshitomi et al., 1998; Kim, 2006); however, the present results show that UCP3 expression, compared to that of $\mathrm{UCP} 2$, is more markedly affected by the state of muscle differentiation. Taken together, the present results indicate that this difference is most likely conferred by different ways their promoter regions respond to the myogenic regulatory factors. Therefore, which binding sites are functional remains to be determined. 
Acknowledgment Dr Dongho Kim was a recipient of a $\mathrm{PhD}$ scholarship awarded by New Zealand Lottery Health.

\section{References}

Arsenijevic, D., Onuma, H., Pecqueur, C., Raimbault, S., Manning, B., Miroux, B., Couplan, E., Alves-Guerra, M., Goubern, M., Surwit, R., Bouilaud, F., Richard, D., Collins, S. and Ricquier, D. (2000) Disruption of the uncoupling protein 2 gene in mice reveals a role in immunity and reactive oxygen species production. Nature Genet. 26, 435-439.

Boss, O., Samec, S., Paoloni-Giacobina, A., Rossier, C., Dulloo, A., Seydoux, J., Muzzin, P. and Giacobina, J. (1997) Uncoupling protein-3: a new member of the mitochondrial carrier family with tissue-specific expression. FEBS Lett. 408, 39-42.

Chevillotte, E., Rieusset, J., Roques, M., Desage, M. and Vidal, H. (2001) The regulation of uncoupling protein-2 gene expression by w-6 polyunsaturated fatty acids in human skeletal muscle cells involves multiple pathways, including the nuclear receptor peroxisome proliferator-activated receptor b. J. Biol. Chem. 276, 10853-10860.

Dedieu, S., Mazères, G., Cottin, P. and Brustis, J. (2002) Involvement of myogenic regulator factors during fusion in cell line $\mathrm{C}_{2} \mathrm{C}_{12}$. Int. J. Dev. Biol. 46, 235-241.

Himms-Hagen, J. and Harper, M. (2001) Physiological role of UCP3 may be export of fatty acids from mitochondria when fatty acid oxidation predominates: an hypothesis. Exp. Biol. Med. 226, 7884.

Horvath, T., Diano, S., Miyamoto, S., Barry, S., Gatti, S., Alberati, D., Livak, F., Lombardi, A., Moreno, M., Goglia, F., Mor, G., Hamilton, J., Kachinskas, D., Horwitz, B. and Warden, C. (2003) Uncoupling protein 2 and 3 influence obesity and inflammation in transgenic mice. Int. J. Obes. 27, 433-442.

Hwang, C. and Lane, M. D. (1999) Up-regulation of uncoupling protein-3 by fatty acid in $\mathrm{C} 2 \mathrm{C} 12$ myotubes. Biochem. Biophys. Res. Commun. 258, 464-469.

Kim, D. (2000) Regulation of expression of uncoupling protein genes, UCP2 and UCP3, in $\mathrm{C} 2 \mathrm{C} 12$ muscle cells. MSc Thesis, University of Otago.

Kim, D. (2006) Regulation of UCP2 and UCP3 gene expression. $\mathrm{PhD}$ Thesis, University of Otago.

Krauss, S., Zhang, C. and Lowell, B. B. (2005) The mitochondrial uncoupling-protein homologues. Nat. Rev. Mol. Cell Biol. 6, 248261.

Larkin, S., Mull, E., Miao, W., Pittner, R., Albrandt, K., Moore, C., Young, A., Denaro, M. and Beaumont, K. (1997) Regulation of the third member of the uncoupling protein family, UCP3, by cold and thyroid hormone. Biochem. Biophys. Res. Commun. 240, 222-227.

Marti, A., Larrate, E., Novo, F., Garcia, M. and Martinez, J. (2001) UCP2 muscle gene transfer modifies mitochondrial membrane potential. Int. J. Obes. 25, 68-74.

Matsuda, J., Hosoda, K., Itoh, H., Son, C., Doi, K., Tanaka, T., Fukanaga, Y., Inoue, G., Nishimaru, H., Yoshimasa, Y., Yamori, Y. and Nakao, K. (1997) Cloning of rat uncoupling protein-3 and uncoupling protein-2 cDNAs: their gene expression in rats fed high-fat diet. FEBS Lett. 418, 200-204.

Murre, C., McCaw, P. S., Vaessin, H., Caudy, M., Jan, L. Y., Jan, Y. N., Cabrera, S. V., Buskin, J. N., Hauschka, S. D., Lassar, A. B.,
Weintraub, H. and Baltimore, D. (1989) Interactions between heterologous helix-loop-helix proteins generate complexes that bind specifically to a common DNA sequence. Cell 58, 537-544.

Nagai, T., Ibata, K., Park, S., Kubota, M., Mikoshiba, K. and Miyawaki, A. (2002) A variant of yellow fluorescent protein with fast and efficient maturation for cell-biological application. Nat. Biotechnol. 20, 87-90.

Nagase, I., Yoshida, S., Canas, X., Irie, Y., Kimura, K., Yoshida, T. and Saito, M. (1999) Up-regulation of uncoupling protein 3 by thyroid hormone, peroxisome proliferator-activated receptor ligands and 9-cis retinoic acid in L6 myotubes. FEBS Lett. 461, 319-322.

Pedraza, N., Rosell, M., Villarroya, J., Iglesias, R., Gonzalez, F. J., Solanes, G. and Villarroya, F. (2006) Development and tissuespecific involvement of peroxisome proliferator-activated receptor-a in the control of mouse uncoupling protein-3 gene expression. Endocrinology 147, 4695-4704.

Schrauwen, P., Hoeks, J., Schaart, G., Kornips, E., Binas, B., Van De Vusse, G., Van Bilsen, M., Luiken, J., Coort, S., Glatz, J., Saris, W. and Hesselink, M. (2003) Uncoupling protein 3 as a mitochondrial fatty acid anion exporter. FASEB J. 17, 2272-2274.

Shimokawa, T., Kato, M., Ezaki, O. and Hashimoto, S. (1998) Transcriptional regulation of muscle-specific genes during myoblast differentiation. Biochem. Biophys. Res. Commun. 246, 287-292.

Solanes, G., Pedraza, N., Inglesias, R., Giralt, M. and Villarroya, F. (2000) The human uncoupling protein-3 gene promoter requires MyoD and is induced by retinoic acid in muscle cells. FASEB $J$. 14, 2141-2143.

Solanes, G., Pedraza, N., Iglesias, R., Giralt, M. and Villarroya, F. (2003) Functional relationship between MyoD and peroxisome proliferator-activated receptor dependent regulatory pathways in the control of the human uncoupling protein-3 transcription. Mol. Endocrinol. 17, 1944-1958.

Solanes, G., Pedraza, N., Calvo, V., Vidal-Puig, A., Lowell, B. B. and Villarroya, F. (2005) Thyroid hormones directly activate the expression of the human and mouse uncoupling protein-3 genes through a thyroid response element in the proximal promoter region. Biochem. J. 386, 505-513.

Son, C., Hosoda, K., Matsuda, J., Fujikura, J., Yonemitsu, S., Iwakura, H., Masuzaki, H., Ogawa, Y., Hayashi, T., Itoh, H., Nishimura, H., Inoue, G., Yoshimasa, Y., Yamori, Y. and Nakao, K. (2001) Up-regulation of uncoupling protein 3 gene expression by fatty acids and agonists for PPARs in L6 myotubes. Endocrinology 142, 4189-4194.

Thompson, M. P. and Kim, D. (2004) Links between fatty acids and expression of UCP2 and UCP3 mRNAs. FEBS Lett. 568, 4-9.

Vidal-Puig, A., Solanes, G., Grujic, D., Flier, J. and Lowell, B. (1997) UCP3: An uncoupling protein homologue expressed preferentially and abundantly in skeletal muscle and brown adipose tissue. Biochem. Biophys. Res. Commun. 235, 79-82.

Vidal-Puig, A., Grujic, D., Zhang, C., Hagen, T., Boss, O., Ido, Y., Szczepanik, A., Wade, J., Mootha, V., Cortright, R., Muoio, D. and Lowell, B. (2000) Energy metabolism in uncoupling protein 3 gene knockout mice. J. Biol. Chem. 275, 16258-16266.

Weigle, D., Selfridge, L., Schwartz, M., Seeley, R., Cummings, D., Havel, P., Kuijper, J. and BeltrandelRio, H. (1998) Elevated free fatty acids induce uncoupling protein 3 expression in muscle. Diabetes 47, 298-302.

Weintraub, H. (1993) The MyoD family and myogenesis: 
redundancy, networks and thresholds. Cell 75, 1241-1244.

Wright, W., Sassoon, D. and Lin, V. (1989) Myogenin, a factor regulating myogenesis, has a domain homologous to MyoD. Cell 56, 607-617.

Yoshitomi, H., Yamazaki, K. and Tanaka, I. (1998) Cloning of mouse uncoupling protein 3 cDNA and 5'-flanking region, and its genetic map. Gene 215, 77-84.

Yoshitomi, H., Yamazaki, K. and Tanaka, I. (1999) Mechanism of ubiquitous expression of mouse uncoupling protein 2 mRNA: control by cis-acting DNA element in 5'-flanking region. Biochem. J. 340, 397-404. 